\title{
USO DE BANDA DENDROMÉTRICA NA DEFINIÇÃO DE PADRÕES DE CRESCIMENTO INDIVIDUAL EM DIÂMETRO DE ÁRVORES DA BACIA DO RIO CUIEIRAS
}

\author{
Roseana Pereira da SILVA ${ }^{1}$; Shozo NAKAMURA²; Celso Paulo de AZEVEDO³; Jeffrey \\ CHAMBERS ${ }^{4}$; Rosana de Miranda ROCHA ${ }^{1}$; Alberto Carlos Martins PINTO ${ }^{5}$; Joaquim dos \\ SANTOS $^{6}$ e Niro HIGUCHI ${ }^{6}$
}

RESUMO: Este trabalho tem como objetivo analisar padrões de crescimento individual de diversas árvores que ocorrem em duas toposseqüências (direçōes Norte-Sul e Leste-Oeste), de uma amostra representativa da floresta de terra-firme na Amazônia Central. Foram selecionados de forma aleatôria, aproximadamente, 300 indivíduos, sendo $150 \mathrm{em}$ cada toposseqüência, distribuídos em mesmas proporções nas três classes topográficas (platồ, encosta e baixio) e nas três classes de diâmetro $(10 \leq \mathrm{DAP}<30 \mathrm{~cm} ; 30 \leq \mathrm{DAP}<50 \mathrm{~cm}$ e DAP $\geq 50 \mathrm{~cm})$. Em cada uma dessas árvores foi instalada uma fita metálica, com extremidades parcialmente sobrepostas e ligadas por uma mola; o avanço de uma das pontas, dentro de uma abertura, representa o crescimento em circunferência, que foi medido com um paquímetro digital. As medições foram realizadas mensalmente ao longo de 19 meses, de junho/1999 a dezembro/2000; neste estudo foram considerados apenas os 12 meses do ano 2000 . O padrão individual de crescimento em diâmetro varia muito com o passar dos meses $(p=0,00)$ e apenas razoavelmente quando os meses são interagidos com as classes de diâmetro ( $p 0,08$ ); por outro lado, há fraca uma evidência $(\mathrm{p}=0,25)$ quando as classes topográficas são acrescentadas na interação anterior e praticamente nenhuma evidência $(\mathrm{p}=0,89)$ quando é analisada a interação meses e classes topográficas. Dentre todas as árvores selecionadas (300 indivíduos), foram mantidas na análise 272 indivíduos. A média do incremento anual em diâmetro, considerando as 272 árvores monitoradas, foi de $1,64 \pm 0,21 \mathrm{~mm}(\mathrm{p}=0,05)$, ficando dentro do intervalo dos incrementos obtidos no BIONTE e FLONA Tapajós, que é de 1,5 a $2 \mathrm{~mm}$ por ano.

PALAVRAS-CHAVE: Floresta amazônica, incremento, manejo florestal.

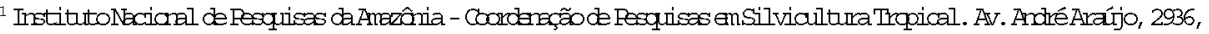
Caixa Fostal 478. FP69.011-970, Meneus, AM, Brasil. Roseana Pereira ca.SilvaE-nail: 1coseainpa.gav lor e Rosana de MirandaRoda Enail: roda @inca.govbror (Bolsistas).

${ }^{2}$ Forestryand Forest Produts Ressarch Institute. P.O. box 16, Tsukiba, NorinKerkyuLanti-rai, Traraki, 305-8587, Japan. Emeil: Inatroaffipri.affic.go.jp (Eesquisadx).

${ }^{3}$ Enibrapa Amazônia Ocidantal - Centro de Pesquisas Agroflorestais da Anezônia Ocidental. Estrada MM 010, km 29, Caixa

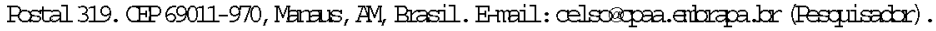

${ }^{4}$ University of Califormia. Ferth Systen.Science. Irvine, CA 926973100 . Emeil: jeffainpa.gor lor (Pesquisedor) .

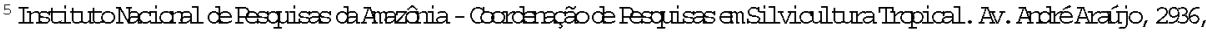
CaixaFostal 478. (FP69.011-970, Manu.s, AM, Brasil. Enail: anartinsairpa.gov bor (Bolsista PCI) .

${ }^{6}$ Institutolbcionel de Pesquisas da Anazânia - Coordenaçãode Pesquisas ensilviaultura Trqpical. Avv. André Araújo, 2936 ,

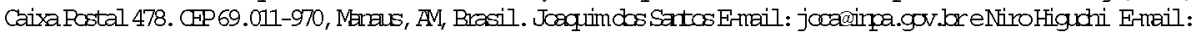
nirozinpa.gribor (Pesquisadores).
} 


\title{
USE OF METALLIC DENDROMETERSFOR INDIVIDUAL DIAMETER GROWTH PATTERNS OF TREES AT CUIEIRAS RIVERBASIN
}

\begin{abstract}
This study dealt with the analysis of individual growth pattern for about 300 trees distributed over two transects (East-West and North-South) measuring 20 by $2500 \mathrm{~m}$, which were stratified by plateau, slope and "baixio", and three diameter at breast height (dbh) classes $(10 \leq \mathrm{dbh}<30$ $\mathrm{cm} ; 30 \leq \mathrm{dbh}<50 \mathrm{~cm}$ e dbh $\geq 50 \mathrm{~cm}$ ). In each tree a metal "dendrometer" band was fixed to the trunk and growth in circumference was measured with a digital caliper. Measurements were carried out for 19 months, from June/1999 to December/2000; for this study only 12 months of year 2000 were considered. Individual growth pattern varied significantly over time $(p=0,00)$, and slightly $(\mathrm{p}=0,08)$ when the interaction months and dbh classes was included; on the other hand, the signal is very weak $(p=0,25)$ when topographical classes were added to the later interaction, and no signal at all $(\mathrm{p}=0,89)$ when the interaction between months and topographical classes were analyzed. Mean annual increment in diameter considering all 272 monitored trees was 1,64 $\pm 0,21$ mm yr-1 $(\mathrm{p}=0,05)$, falling within the interval estimated for BIONTE and Tapajos National Forest which are 1,5 and $2 \mathrm{~mm}$ per year, respectively.
\end{abstract}

KEYWORDS: Amazon forest, increment, forest management.

\section{INTRODUÇÃO}

Grande parte dos estudos publicados sobre crescimento e rendimento de florestas tropicais enfatiza apenas a escala de ecossistema ou de povoamento florestal, sem levar em consideração o padrão de crescimento individual das espécies. Na literatura florestal, os índices de produtividade (incremento periódico anual em volume de madeira) de florestas manejadas em diferentes sítios florestais da Ásia e África variam de 2 a 4 $\mathrm{m}^{3} /$ ha/ano, de acordo com Wadsworth (1987) e Leslie (1987). Na Amazônia brasileira, nos sítios de Santarém (FLONA Tapajós) e Manaus (ZF2, conhecido como BIONTE), o incremento periódico anual em volume, dez anos após a exploração florestal, pode ser estimado em $5 \mathrm{~m}^{3} /$ ha/ano (Silva et al., 1996 e Higuchi et al., 1985). Esses índices, geralmente, são baseados em medições contínuas em parcelas permanentes.
Na Amazônia, praticamente não há estudos de correlação entre padrões de crescimento individual e fatores climáticos. As poucas informações existentes sobre padrões de crescimento individual de espécies arbóreas tropicais, são sobre poucas espécies e usadas apenas para comparações com aquelas manejadas (Manokaran \& Kochummen, 1993; Milton etal., 1994; Herwitz \& Young, 1994; Condit et al., 1995; Silva et al., 1996; Poels et al., 1998 e Finegan et al., 1999).

$\mathrm{O}$ entendimento acerca da dinâmica e mecanismos envolvidos no crescimento e desenvolvimento de espécies arbóreas, pode ajudar a explicar muitos dos questionamentos levantados quando se pretende conciliar produçāo e conservação. No caso específico de manejo florestal, esse entendimento é fundamental em importantes tomadas de decisão, como (i) escolha das espécies que podem ser exploradas; (ii) 
escolha das espécies que devem ser protegidas; (iii) projeção mais precisa do ciclo de corte e (iv) prescrição de tratamentos silviculturais.

A estrutura da vegetação determina muita das características das paisagens, nas quais outros organismos vivem e se desenvolvem, incluindo o ser humano (Walter, 1979). Da mesma forma, as plantas respondem e são dependentes desses mesmos fatores e organismos. Assim, para estabelecer medidas de conservação e proteção de ecossistemas tão complexos e heterogêneos como os da Amazônia, é preciso também entender como as plantas respondem aos fatores climáticos, edáficos e bióticos e vice-versa.

Entre os fatores de interação obrigatória com as plantas, a água é a substância inorgânica mais importante e está presente nas plantas em grande quantidade. A precipitação é a maior fonte de umidade do solo e principal fonte de água que alcança a árvore. Quando o solo seca, a fotossíntese gradualmente diminui aumentando a resistência à fixaçāo do $\mathrm{CO}^{2}$ por causa do fechamento dos estômatos. Tribuzy (1998) afirma que o excesso de água, tanto quanto o estresse hídrico, dificulta a fotossíntese e o crescimento dos vegetais.

As árvores processam seu crescimento em diâmetro em larga escala às expensas dos produtos correntes da fotossíntese, sendo sensível às condiçōes do meio, e especialmente às disponibilidades hídricas (Ferri, 1979). Entretanto, a determinação do balanço hídrico na região amazônica tem sido dificultada por causa da falta de continuidade nas medidas de precipitação; pela omissão da água armazenada no solo e a localização das estações concentradas em margens dos principais rios. Para Santos (1968), a disponibilidade de água no solo constitui, sem dúvida, um dos fatores preponderantes no levantamento das possibilidades e limitações climáticas de um determinado local, mas, torna-se também necessário que sejam levados em consideração fatores relacionados com a evapotranspiração. Como a evapotranspiração é um fenômeno climático, que só se realiza às custas do consumo de energia e como essa energia, na natureza, provém da radiação solar, deduz-se que um povoamento florestal exerce grande influência sobre a variação da evapotranspiração.

O acréscimo diamétrico varia significativamente entre e dentro das espécies arbóreas e de acordo com a idade, estações do ano e condições microclimáticas. Muitas espécies de florestas tropicais, geralmente, apresentam comportamentos bastante diferenciados, estando sempre associada às respostas individuais de cada espécie, cada família, ou até mesmo cada indivíduo observado. Nas análises existentes sobre a relação entre a umidade do solo e o crescimento, considera se que este último não è um fator diretamente controlado pela umidade do solo, mas pelo equilíbrio hídrico da planta, que por sua vez, é regulado pelas intensidades relativas de absorçāo e de transpiração; sendo, por esta razāo, afetado tanto pelas condiçōes de umidade do solo como pelas atmosféricas (Ferri, 1979).

Além dos índices de produtividade do povoamento florestal e do entendimento dos processos ecofisiológicos que explicam o funcionamento (resposta à intervençāo) do ecossistema manejado, o manejo florestal sustentável requer informaçōes sobre o padrāo de crescimento individual das espécies. As medidas de crescimento diamétrico têm sido freqüentemente usadas nos estudos que examinam a resposta de crescimento natural das árvores ou mudanças antropogênicas do ambiente (Lea et al., 1979; Day, 1985; Conner \& Day, 1992).

As informações sobre crescimento individual poderiam ser facilmente tiradas das parcelas permanentes, tendo em vista que a principal variável para este tipo de trabalho, "o diâmetro" (normalmente à altura do peito, DAP), é a única variável comum em todas as parcelas de todos os sítios de pesquisa. Entretanto, no 
caso do crescimento do povoamento, essas informaçōes permanecem nos bancos de dados das instituições responsáveis pelas parcelas permanentes.

No Sudeste Asiático, Manokaran \& Kochummen (1993) apresentam os resultados de incremento de 48 espécies florestais, com base em parcelas instaladas em dois sítios diferentes na Malásia Peninsular, em 1947 e 1949 e avaliadas em 1985. As estimativas dos incrementos médios anuais em diâmetros foram: de 0,4 a 1,6 mm/ano nas espécies de sub-bosque; 0,5 a $3,5 \mathrm{~mm} /$ ano nas principais espécies do dossel; 2,7 a $4,5 \mathrm{~mm} /$ ano nas espécies emergentes; de 1,5 a 4,5 mm/ano nas espécies pioneiras.

Além do trabalho de Manokaran e Kochummen (1993), há na literatura, apenas registros de estudos de crescimento individual de mudas, realizados por Chim \& On (1973) e de Turner (1990), respectivamente na Malásia Peninsular e Sabah.

$\mathrm{Na}$ América Tropical, principalmente América Central, muitos estudos sobre crescimento individual de árvores têm sido implementados ao longo das duas últimas décadas, dando maior ênfase ao entendimento da biologia e ecologia das espécies do que em direção ao manejo florestal. Os dois principais sítios de pesquisa da América Central sāo Estaçāo Biológica La Selva, na Costa Rica e a Ilha de Barro Colorado, no Panamá.

Clark \& Clark (1987) observaram a ecologia de populações e distribuição de micro-habitat de uma única espécie, Dipteryx panamensis, na Estação La Selva, Costa Rica. Os indivíduos pósgeminados de 7 meses a 5 anos (plântulas) foram estudados em uma parcela de 1 ha e os indivíduos não plântulas foram observados em uma parcela de 4 ha onde a estrutura da população foi determinada por meio de inventário completo. Esses autores relatam que à medida que ocorre $o$ crescimento diamétrico, a taxa de mortalidade diminui, tendo sido observado que dos 105 indivíduos com DAP $\geq 10 \mathrm{~cm}, 104$ sobreviveram após 2 anos de observações. $\mathrm{O}$ crescimento em diâmetro dos indivíduos com DAP $<10 \mathrm{~cm}$ foi menor que $1 \mathrm{~mm} / \mathrm{ano}$, enquanto nos indivíduos maiores, com DAP entre 10 a $70 \mathrm{~cm}$, o crescimento diamétrico foi em média 5 a $8 \mathrm{~mm} /$ ano. $\mathrm{O}$ crescimento relativamente rápido dos indivíduos juvenis e adultos sugere que Dipteryx panamensis está se regenerando in situ e, para descrever a regeneração de florestas tropicais úmidas, tornamse indispensáveis o monitoramento do ambiente e o acompanhamento das medidas de crescimento em longo prazo.

No mesmo sítio de pesquisa, Clark \& Clark (1999) divulgaram resultados de uma análise de 11 anos (1985-1995) em parcelas permanentes, medidas anualmente, instaladas para acompanhar o crescimento de 7 espécies não pioneiras (Minquartia guianensis, Lecythis ampla, Hymenolobium mesoamericanum, Simarouba amara, Dipteryx panamensis, Pithecollobium elegans e Hyeronima alchorneoides) e 2 pioneiras (Cecropia obtusifolia e C. insignis). Para todas as 9 espécies, os autores concluíram que os padrões de crescimento em diâmetro são altamente dependentes do tamanho da árvore e que as pioneiras têm crescimento superior ao das nāo pioneiras até atingir o $\mathrm{DAP}=30 \mathrm{~cm}$. Outras conclusões são: nas classes inferiores (até $10 \mathrm{~cm}$ em DAP), as espécies não pioneiras apresentam padrōes parecidos e baixos, menores que $5 \mathrm{~mm} /$ ano, e que o crescimento nāo está correlacionado com o tamanho da árvore; nas classes superiores (DAP $\geq 20 \mathrm{~cm}$ ), o crescimento é maior, podendo atingir de 7 a $8 \mathrm{~mm} /$ ano; a capacidade de crescimento nāo declina em direçāo ao zero, conforme a espécie se aproxima ao tamanho máximo. Por último, foi concluído também que os resultados obtidos indicam que os pressupostos 
dos modelos atuais de dinâmica da floresta não se aplicam àquele sítio de pesquisa (Clark e Clark, 1999).

Segundo Keeland \& Sharitz (1993), o uso de bandas (ou fitas) dendrométricas permanentes para monitorar o crescimento em diâmetro foi introduzido por Hall (1944), em floresta temperada, tendo desde o início, um uso satisfatório e convincente em medições repetidas. Desde então, essas fitas têm sido amplamente utilizadas em florestas temperadas, o que não se repete em florestas tropicais. Na Amazônia brasileira, por exemplo, há registros de utilização dessas fitas apenas em Santarém e em Manaus, mas sem nenhum trabalho científico publicado na literatura.

As vantagens observadas na utilização de fitas dendrométricas são: (1) facilidade na instalaçāo e leitura; (2) custo baixo e (3) nāo acarretam danos no caule e no câmbio (Keeland \& Sharitz, 1993). Por outro lado, a principal desvantagem observada por Bower \& Blocker (1966) e confirmada por Cameron \& Lea (1980), é o fato que no primeiro ano de observações, as mediçōes em bandas tendem a subestimar o crescimento em diàmetro. Entretanto, Day (1985), apud Keeland \& Sharitz (1993) concluiu que em regiōes onde as estaçōes do ano sāo bem definidas, a subestimaçāo das medidas do diâmetro pode ser atribuída à falta aparente de crescimento, que provoca o relaxamento dos encaixes (molas) de instalação das bandas.

Há também dendrômetros automáticos de alta precisão que estão sendo utilizados em florestas temperadas, principalmente quando o objetivo é analisar o relacionamento entre a fenologia e o crescimento individual da árvore. Tabuchi e Takahashi (1998) relatam sobre a experiência com esse tipo de dendrômetro, tipo $\mathrm{Hi}$-Fi, instalado em espécies caducifólias no Norte do Japão, Hokkaido; as medidas de mudanças na circunferência do tronco foram tomadas de hora em hora, durante a estação de crescimento das plantas. Essa primeira experiência com esse tipo de equipamento, durante o período de abrilagosto, foi considerada promissora pelos autores, porque permite descrever o padrão de crescimento individual, detalhadamente e com muita precisão, e relacioná-lo com dados de disponibilidade de água e outras variáveis climatológicas.

Este trabalho tem como objetivo analisar padrōes de crescimento individual de diversas árvores que ocorrem em duas toposseqüências (direções Norte-Sul e Leste-Oeste), estratificadas em platô, encosta e baixio, de uma amostra representativa da floresta de terra-firme da Amazônia Central. O incremento em diâmetro será correlacionado com precipitação, classes topográficas e classes de diâmetro. Será ainda analisado o padrāo de crescimento em funçāo tempo e classe de diâmetro, bem como tempo e classe topográfica.

\section{METODOLOGIA}

\section{Caracterização da Área de Estudo}

O trabalho foi realizado na Estação Experimental de Silvicultura Tropical do INPA (Núcleo ZF2), localizada a $90 \mathrm{~km}$ ao norte de Manaus, nos dois transectos, sentidos nortesul e leste-oeste. Esses transectos foram descritos por Silva (2001), onde já foram executados estudos sobre a vegetação (Higuchi et al., 1998) e solos (Ferraz et al., 1998), ambos considerando separadamente os estratos platô, encosta e baixio.

De acordo com Ferraz et al. (1998), os solos dos platôs são de textura argilosa; nas encostas, variam de argilo-arenoso (próximo aos platôs) e areno-argilosos (próximos aos baixios) e nos baixios, são de textura arenosa. Os mesmos autores classificam os solos nas áreas dos 
transectos em três tipos: (1)-Latossolo Amarelo nos platôs; (2)-Podzólicos Vermelho-Amarelo, nas encostas; e (3)-Arenossolos hidromórficos nos baixios. O transecto L-O apresentou uma segunda área de baixio, onde foi observado um podzol. Ao longo das duas topossequiências os solos são muitos ácidos, e nas depressões, apresentam maiores concentrações de cargas negativas.

Essa área é coberta por floresta tropical úmida densa de terra firme, típica da parte central da região amazônica (Higuchi et al., 1997). Em área vizinha, aproximadamente $10 \mathrm{Km}$ à leste da área de estudo, através de inventário florestal, Higuchi et al. (1985), encontraram numa área de 96 ha, 14.922 indivíduos com $\mathrm{DAP} \geq 25 \mathrm{~cm}$, distribuídos em 51 famílias botânicas diferentes, com 409 espécies para 206 gêneros. As espécies mais abundantes foram castanha jarana (Lecythis sp.), inharé (Helicostylis podogyne Ducke) e uxi (Endopleura uchi Huber).

Segundo classificação de Köppen, o tipo climático é Am. O clima é quente e úmido durante praticamente $o$ ano inteiro, apresentando umidade relativa média muito alta, variando de $84 \%$ a $90 \%$. Os valores mais altos são observados nos meses de dezembro a maio.

\section{Coleta de Dados}

Inicialmente foram sorteadas aleatoriamente, 300 árvores de um banco de dados do trabalho de Higuchi et al. (1998), sendo 150 árvores em cada transecto. Essas árvores foram agrupadas nas seguintes classes diamétricas: $10 \leq \mathrm{DAP}<30 ; 30 \leq \mathrm{DAP}<50$ e $\mathrm{DAP} \geq 50 \mathrm{~cm} \mathrm{e}$ distribuídas na medida do possível, em mesmo número de indivíduos, considerando ainda a estratificação topográfica (platô, encosta e baixio) ao longo de cada transecto $(20 \times 2500 \mathrm{~m})$.

Em cada árvore sorteada foi instalada uma "banda dendrométrica" (Figura 1) confeccionada de forma manual, que mede a expansão do crescimento do tronco por meio do deslocamento provocado por uma mola que se desloca a medida que ocorre o crescimento do fuste. As medidas das variações de crescimento foram obtidas utilizando um paquímetro digital, com precisão de centésimos de milímetro. As aberturas são sobrepostas em torno do fuste, tendo as extremidades da banda interligadas por uma mola que promove o deslocamento e o conseqüente movimento na posiçāo das aberturas, fornecendo a variaçāo das medidas tomadas mensalmente.

\section{Delineamento Estatístico}

Para atender os objetivos específicos do projeto, quatro diferentes testes estatísticos foram realizados: (a) testes de correlaçōes, (b) comparaçāo de médias entre transectos, (c) comparaçāo de médias entre classes topográficas e (d) comparação de médias combinadas, classes topográficas e tempo.

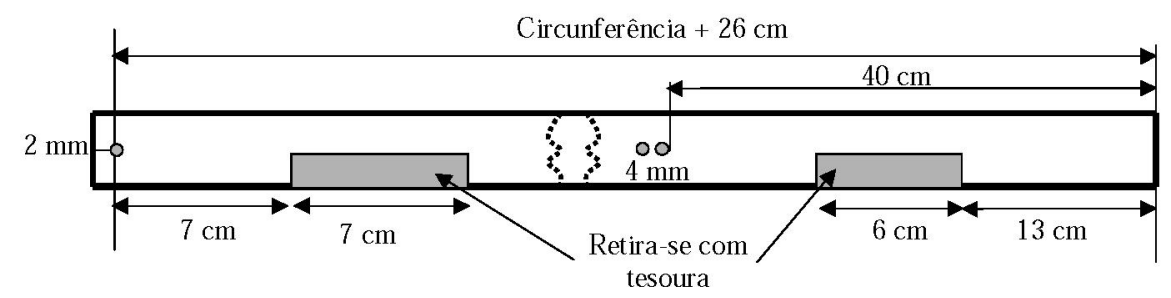

Figura 1. Esquema para fabricação das bandas dendrométricas. 


\section{(a) Correlações}

De acordo com Weiss \& Hasset (1982) e Freund \& Walpole (1987), as seguintes correlações foram testadas: (i) Incremento corrente mensal (ICM) e precipitação; (ii) ICM e classes de diâmetro e (iii) ICM e classes topográficas.

\section{(b) Comparação de médias entre transectos}

De acordo com Weiss \& Hasset (1982) e Freund \& Walpole (1987), foi testada a seguinte hipótese nula: Não há diferença em ICM em diâmetro entre os transectos orientados nos sentidos Norte-Sul e Leste-Oeste, ou seja, a média do ICM do transecto $1, \mu_{1}$, é igual a média do transecto $2, \mu_{2}$.

\section{(c) Comparação de médias entre classes topográficas}

De acordo com Weiss \& Hasset (1982) e Freund \& Walpole (1987), foi testada a seguinte hipótese nula: Não há diferença em ICM em diâmetro entre as classes topográficas (platô, encosta e baixio), ou seja, a média do ICM do platô $1, \mu_{1}$, é igual a média da encosta, $\mu_{2}$, que é igual a do baixio, $\mu_{3}$.

(d) Comparação de médias combinadas, classes topográficas (CT) e tempo (T) Medições repetidas

Este teste foi levado a cabo de acordo com von Ende (1993), considerando três condiçōes topográficas (platô, encosta e baixio) e 12 medições mensais do crescimento em diâmetro. $\mathrm{O}$ objetivo foi de verificar se o crescimento em diâmetro é influenciado pelas diferentes classes topográficas, com o passar do tempo. A análise foi feita com base nos ICMs médios das classes de diâmetro, que foram ainda subdivididas em classes menores com intervalos de $5 \mathrm{~cm}$, totalizando, dessa maneira, 432 células (12 classes de DAP x 3 CTs $x 12$ meses). A hipótese nula testada foi: O padrāo de crescimento individual nāo depende das classes topográficas (CT) e nem do tempo ( $\mathrm{T}$ ). Os valores de $\mathrm{F}$ foram substituídos por G-G e H-F, que sāo probabilidades ajustadas por GreenhouseGeisser e Huynh-Feldt, respectivamente.

\section{RESULTADOS E DISCUSSÃO}

As bandas dendrométricas foram instaladas em junho de 1999, quando foi registrada a primeira mediçāo. As mediçōes foram feitas mensalmente sempre entre os dias 25 e 30 de cada mês. De acordo com a literatura, geralmente as três primeiras medições estão dentro da fase de ajuste da banda ao fuste e, por essa razão, devem ser descartadas da análise; o quê também foi observado neste estudo. Para este estudo específico foram consideradas apenas as doze medições mensais feitas no ano de 2000, aumentando a segurança em relação a fase de ajuste das bandas, de três para sete meses.

A principal variável ambiental utilizada para comparações com o crescimento individual foi a precipitação. Os dados de precipitação diária foram coletados com auxílio de um pluviômetro instalado na própria área de estudo, na ZF-2. A Tabela 1 apresenta as informações consolidadas mensalmente da ZF-2, assim como as precipitaçōes médias mensais da série histórica 1980-2000 obtidas da estação meteorológica da EMBRAPA, localizada, aproximadamente, $50 \mathrm{~km}$ a sudeste da área de estudo. 
Tabela 1. Médias de precipitação pluviométrica mensal $(\mathrm{mm})$ da série histórica $1980-2000$, coletados na EMBRAPA, para efeito de comparação com os dados coletados em 2000 pelo pluviômetro da ZF-2.

\begin{tabular}{lcc}
\hline meses & $\mathbf{1 9 8 0 - 2 0 0 0}$ & ZF2-2000 \\
\hline Janeiro & 293,2 & 254,0 \\
Fevereiro & 298,2 & 281,2 \\
Março & 306,2 & 288,8 \\
Abril & 323,6 & 736,4 \\
Maio & 270,7 & 521,9 \\
Junho & 168,1 & 206,2 \\
Julho & 105,0 & 132,1 \\
Agosto & 114,6 & 125,5 \\
Setembro & 118,4 & 212,3 \\
Outubro & 156,9 & 189,0 \\
Novembro & 207,5 & 302,0 \\
Dezembro & $\mathbf{2 4 7 , 7}$ & 241,9 \\
\hline Precip. Anual & $\mathbf{2 6 1 0 , 1}$ & $\mathbf{3 4 9 1 , 3}$ \\
\hline
\end{tabular}

Uma análise expedita da série histórica 1980-2000 do CPAA-EMBRAPA mostra que o intervalo de confiança para a precipitação média anual é $2.610 \pm 124 \mathrm{~mm}(\mathrm{p}=0,05)$. Na área de estudo, a precipitação do ano 2000 foi de 3.491 $\mathrm{mm}$, caindo fora do intervalo de confiança da série histórica da EMBRAPA. De um lado, este fato é extremamente positivo porque, num ano atípico em relação à precipitação, foi determinado o padrão de crescimento individual; por outro lado, é muito arriscado fazer extrapolações ou projeções nessas condições. Isso quer dizer que toda a análise envolvendo este estudo se refere aos sinais de padrões individuais de crescimento em relação às classes topográficas e de diâmetro, adiando as projeções para quando for completado pelo menos mais um ano de monitoramento.

De um modo geral, a sazonalidade da região é caracterizada apenas por duas estaçōes, a estação chuvosa que ocorre de dezembro-maio e a seca que vai de junho-novembro (Ribeiro \& Adis, 1984). Mesmo num ano atípico, é possível perceber a diminuição de chuvas entre os meses de junho e novembro, que é uma característica da regiāo (Tabela 1 ).

\section{Caracterização dos indivíduos arbóreos estudados}

Na última medição considerada neste estudo, dezembro/2000, o número total de árvores com bandas era de 272. A mortalidade natural durante o período observado foi de 6 árvores, em torno de $2 \%$ ao ano, que é uma taxa aceitável para a região de Manaus (Higuchi et al., 1997). Além da mortalidade natural, houve necessidade de substituições de bandas de algumas árvores, que foram excluídas do presente trabalho. As principais causas para as substituiçōes foram a formaçāo de cupinzeiros que alteraram o ajuste das molas e a quebra do conjunto "fita e mola" da banda dendrométrica. Os dados analisados neste estudo foram distribuídos de acordo com a Tabela 2, procurando atribuir, aproximadamente, o mesmo número de árvores nas três classes topográficas $\mathrm{e}$ ao longo de toda a extensão de cada transecto.

A Tabela 3 apresenta as três famílias botânicas com maior número de indivíduos dentro da amostra selecionada, que foram Sapotaceae, Lecythidaceae e Caesalpiniaceae. Esse resultado está de acordo com as observaçōes feitas nos 
Tabela 2. Distribuição do número de árvores com dados analisados.

Transectos Norte-Sul e Leste-Oeste

\begin{tabular}{|c|c|c|c|}
\hline \multicolumn{2}{|c|}{ Classe diamétrica } & \multicolumn{2}{|c|}{ Classe topográfica } \\
\hline $10<\mathrm{DAP} \leq 30$ & 89 & Platô & 105 \\
\hline $30<\mathrm{DAP} \leq 50$ & 101 & Encosta & 76 \\
\hline $\mathrm{DAP} \geq 50 \mathrm{~cm}$ & 82 & Baixio & 91 \\
\hline
\end{tabular}

estudos de Higuchi et al. (1998), que consideraram mais de 6.000 árvores nos dois transectos. Dessa forma, além da proporcionalidade em relação à orientação dos transectos, classes topográficas e classes de diâmetro, houve também a proporcionalidade na distribuição dos indivíduos nas famílias botânicas, ainda que fortuitamente. dois sítios, as medições são feitas anualmente sem bandas dendrométricas em mais de 2.000 indivíduos.

As espécies com o menor (negativo) e o maior incremento anual foram maueira (Erisma bicolor Ducke, Vochysiaceae) e louro fofo (Ocotea immersa van der Werff, Lauraceae), respectivamente. Das 272 árvores com bandas

Tabela 3. Famílias botânicas mais abundantes.

\begin{tabular}{lcc}
\hline Família & $\begin{array}{c}\text { Número de } \\
\text { Árvores }\end{array}$ & $\begin{array}{c}\text { Abundância } \\
\text { Relativa (\%) }\end{array}$ \\
\hline Sapotaceae & 44 & 16,1 \\
Lecythidaceae & 42 & 15,4 \\
Caesalpinoideae & 20 & 7,3 \\
\hline
\end{tabular}

\section{Incremento dos indivíduos estudados}

A média do incremento anual, considerando todos os indivíduos $(\mathrm{n}=272)$ foi igual a $1,64 \mathrm{~mm}$, com intervalo de confiança de 1,43 a $1,85 \mathrm{~mm}(\mathrm{p}=0,05)$ e valores mínimo e máximo de $-0,48 \mathrm{~mm}$ e $11,41 \mathrm{~mm}$, respectivamente. A estimativa do incremento anual deste trabalho $(1,64 \mathrm{~mm})$ está dentro do intervalo de incrementos obtidos na área do BIONTE (Higuchi et al., 1997) e na Floresta Nacional do Tapajós (Silva et al., 1996), respectivamente $1,5 \mathrm{~mm}$ e $2,0 \mathrm{~mm}$ por ano. Grande parte dessa variação pode ser atribuída à quantidade de chuva no ano 2000; outra parte pode ser por causa da forma de medição dos DAP's e ao tamanho da amostragem. Nos outros dendrométricas, 42 (9 espécies listadas e 33 não listadas) apresentaram incremento anual igual a zero; 117 (45 listadas) apresentaram em média incremento de $0,64 \mathrm{~mm}$; 69 (25 listadas) apresentaram incremento médio de $2,23 \mathrm{~mm}$ e 44 (21 listadas) apresentaram incremento médio de $4,77 \mathrm{~mm}$.

Com apenas um ano de observações, é muito difícil definir estratégias silviculturais com base no padrão individual de crescimento em diâmetro. Na Tabela 4 são apresentados os incrementos médios e respectivos coeficientes de variação de algumas espécies selecionadas, que tinham pelo menos 4 indivíduos. Dois extremos são óbvios; Goupia glabra Aubl. (cupiúba) com CV $=38 \%$ e Hevea guianensis Aubl. (seringa vermelha) $\operatorname{com~CV}=431 \%$. No 
primeiro caso, os resultados deste trabalho indicam que a cupiúba dispensa tratamento silvicultural, para favorecer o seu crescimento, porque essa espécie tem um bom crescimento independentemente de sua posiçāo e associaçāo botânica. No segundo caso, ao contrário, os resultados indicam a necessidade de tratamentos, apesar de não haver garantias de respostas positivas à intervenção.

As espécies Eschweilera coriacea (DC.) Mart. ex Berg. (matamatá), Protium altsonii Sandwith (breu), Scleronema micranthum
Ducke (cardeiro) e Virola calophylla Warb. (ucuúba), todas espécies listadas, apresentaram incrementos anuais, em condições naturais, bem acima das médias do BIONTE e do Tapajôs, respectivamente $2.3 \mathrm{~mm}, 1,8 \mathrm{~mm}, 2,1 \mathrm{~mm}$ e 2,4 $\mathrm{mm}$ por ano. Com base nos seus coeficientes de variaçāo, há indicaçōes que sāo espécies que apresentam alguma elasticidade, ou seja, essas espécies devem responder favoravelmente aos tratamentos silviculturais.

Tabela 4 . Coeficiente de Variação (CV), em relação ao incremento anual em diâmetro de algumas espécies arbóreas selecionadas (com no mínimo $n=4$ ).

\begin{tabular}{|c|c|c|c|c|c|}
\hline Nome científico & Família & Nome Comum & $\begin{array}{c}\text { Média } \\
(\mathrm{mn})\end{array}$ & $\begin{array}{c}\text { Desvio } \\
(\mathrm{mn})\end{array}$ & $\begin{array}{l}\text { Cv } \\
(\%)\end{array}$ \\
\hline $\begin{array}{l}\text { Chrysophyllum } \\
\text { ucuquirana-branca Aub. } \\
\text { \& Pell. }\end{array}$ & Sapotaceae & ucuquirana & 1,29 & 1,07 & 83 \\
\hline $\begin{array}{l}\text { Ecclinusa guianensis } \\
\text { Eyma }\end{array}$ & Sapotaceae & abiurana & 1,33 & 1,67 & 125 \\
\hline $\begin{array}{l}\text { Eperua glabriflora } \\
\text { (Ducke) } \\
\text { R.S.Cowan }\end{array}$ & Caesalpinioideae & muirapiranga & 0,90 & 1,10 & 123 \\
\hline $\begin{array}{l}\text { Eschweilera coriacea } \\
\text { (DC.) } \\
\text { Mart. ex Berg. }\end{array}$ & Lecythidaceae & matamata & 2,39 & 1,86 & 78 \\
\hline $\begin{array}{l}\text { Eschweilera } \\
\text { cyathiformis } \\
\text { S.A.Mori }\end{array}$ & Lecythidaceae & cast.vermelha & 2,05 & 1,46 & 71 \\
\hline Goupia glaba Aubl. & Celastraceae & cupiúba & 1,77 & 0,68 & 38 \\
\hline Hevea guianensis Aubl. & Euphorbiaceae & seringa & 0,04 & 0,16 & 431 \\
\hline $\begin{array}{l}\text { Micrandra siphonioides } \\
\text { Benth. }\end{array}$ & & & & & \\
\hline $\begin{array}{l}\text { Micropholis guyanensis } \\
\text { (A.DC) Pierre }\end{array}$ & $\begin{array}{l}\text { Euphorbiaceae } \\
\text { Sapotaceae }\end{array}$ & $\begin{array}{l}\text { seringarana } \\
\text { rosada brava }\end{array}$ & $\begin{array}{l}3,58 \\
1,58\end{array}$ & $\begin{array}{l}1,98 \\
2,15\end{array}$ & $\begin{array}{r}55 \\
137\end{array}$ \\
\hline $\begin{array}{l}\text { Protium altsonii } \\
\text { Sandwith }\end{array}$ & Burseraceae & breu & 1,84 & 1,22 & 66 \\
\hline $\begin{array}{l}\text { Scleronema Micranthum } \\
\text { Ducke }\end{array}$ & Bombacaceae & cardeiro & 2,13 & 1,88 & 89 \\
\hline Virola calophylla Warb. & Myristicaceae & ucuúba & 2,46 & 2,06 & 84 \\
\hline
\end{tabular}




\section{Padrões de crescimento em diâmetro}

Apesar do período observado ter sido atípico em relação à precipitação anual, o padrão de crescimento em diâmetro é caracterizado pela diminuição do incremento nos meses mais secos do ano, de maio a setembro. Independentemente de como é analisado, considerando as classes topográficas e de diâmetro juntas (Figura 2) ou separadamente (Figuras 2a, 2b e 2c), o padrão é o mesmo. A correlaçāo entre precipitaçāo e incremento corrente mensal (ICM) em DAP foi positiva, $\mathbf{r}=0,398$, porém apenas significante a $20 \%$. O sinal positivo indica que há uma tendência dos maiores ICMs estarem associados às maiores precipitaçōes ou, os menores ICMs estarem associados com as menores precipitações.

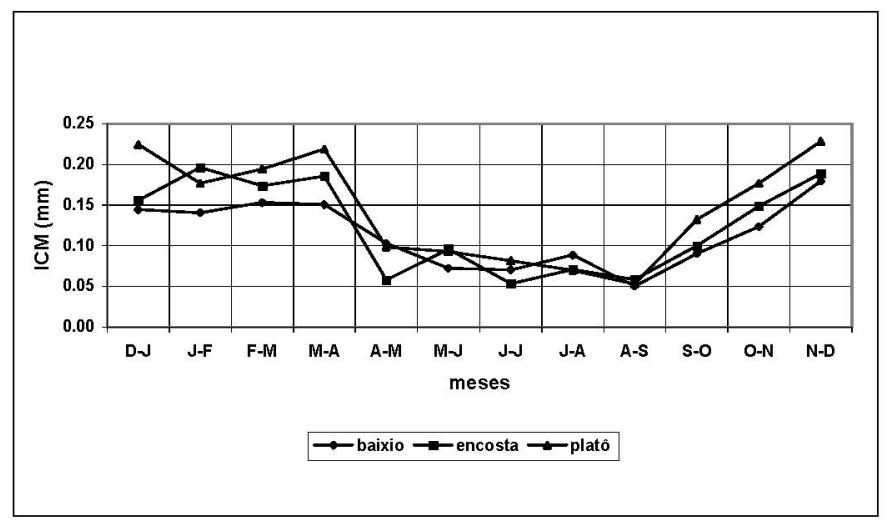

Figura 2. Incremento Corrente Mensal (ICM) dos DAPs das diferentes classes topográficas em relação aos meses do ano 2000 .

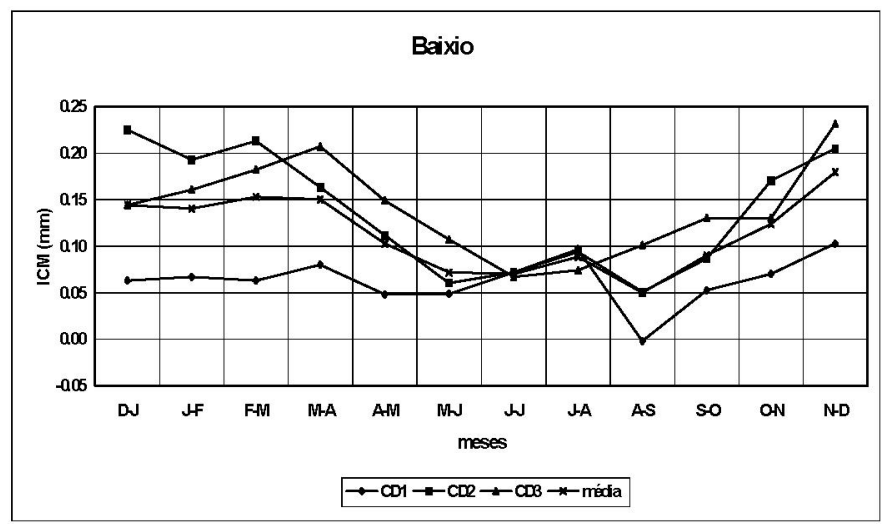

Figura 2a. Incremento Corrente Mensal (ICM) de diferentes classes de DAP em relação aos meses do ano 2000 - Baixio. 


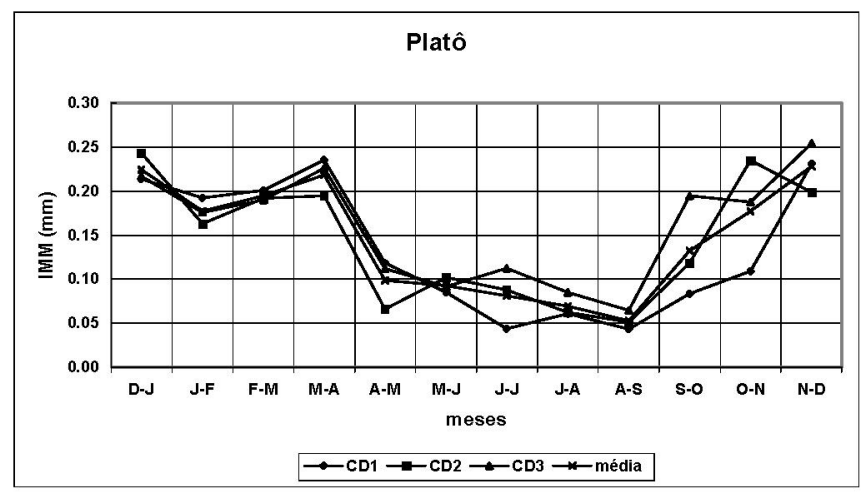

Figura 2b. Incremento Corrente mensal (ICM) de diferentes classes DAP em relação aos meses do ano 2000 - Encosta.

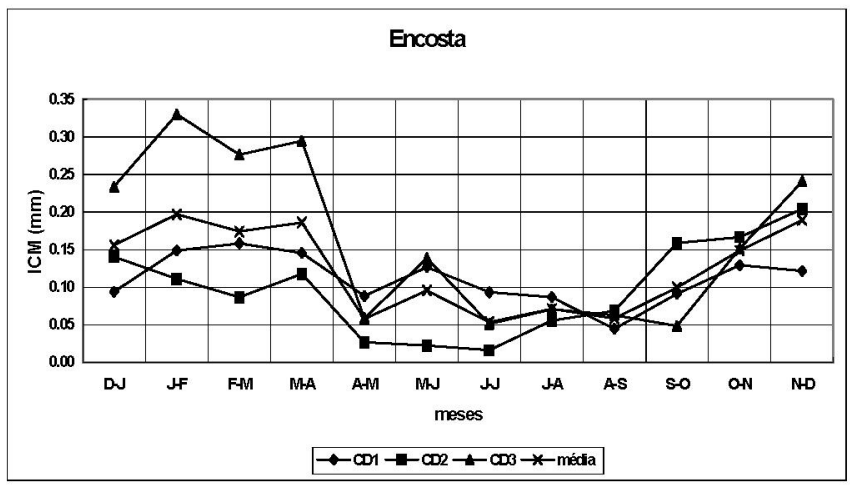

Figura 2c. Incremento Corrente Mensal (ICM) de diferentes classes de DAP em relação aos meses do ano 2000 - Platô.

Para os padrões usados na Engenharia Florestal, principalmente, quando se correlaciona volume de madeira e variáveis independentes como DAP e altura, um valor de $r=0,398$ pode ser considerado um coeficiente muito baixo. No entanto, num estudo exploratório como este, $r=0,398$ não pode ser negligenciado e nem $p=0,20$ pode ser considerado como verdade absoluta para inferir sobre a correlação das variáveis consideradas. Quando correlacionado com a série histórica da EMBRAPA, o coeficiente muda para $\mathrm{r}=0,886$ $(p=0,001)$. Esses diferentes coeficientes de correlação podem indicar que a "quantidade" de chuva pode nāo ser o fator mais importante e sim, como a chuva é distribuída ao longo do mês ou da estação do ano (estação seca e chuvosa).

\section{Incremento corrente mensal (ICM) e classes de diâmetro:}

Para executar essa análise, os ICMs foram grupados (estimando a média posteriormente) 
proporcionalmente à freqüência de cada classe de diâmetro, de modo a reduzir as tendências provocadas pela forma de J-invertido dos dados observados. Dessa forma, a correlação entre ICM e classes de diâmetro foi feita com base em $n=27$.

O coeficiente de correlação Pearson ( $r$ ) foi de 0,584 , positivo e altamente significante $(\mathrm{p}=$ 0,00098), evidenciando que os maiores ICMs estão relacionados com os maiores diâmetros. Essa tendência está representada graficamente na Figura 3. Também nesse caso, o padrão de crescimento, durante $\mathbf{o}$ ano observado, é igual nas três classes uma classe diamétrica é estatisticamente diferente das demais e que, a maior classe diamétrica (DAP $\geq 50 \mathrm{~cm})$ é significantemente $(\mathrm{p}=0,05)$ diferente e maior do que as outras duas classes menores.

Na Figura 3 é possível observar ainda que, ao contrário do que é esperado, as árvores de maior porte diamétrico apresentaram maiores incrementos. Isso pode ser em decorrência da maior atividade fotossintética, uma vez que essas árvores, de acordo com Hubbell et al. (1999), em sua grande maioria, ocupam o dossel da floresta,

Tabela 5. Variação entre as classes de diâmetro.

\begin{tabular}{lccccc}
\hline Fonte de variação & $\mathrm{SQ}$ & $\mathrm{GL}$ & $\mathrm{MQ}$ & $\mathrm{F}$ & $\mathrm{P}$ \\
\hline Classes diamétricas & 0.03826 & 2 & 0.01913 & 4.16743 & 0.02991 \\
Erro & 0.09640 & 21 & 0.0549 & & \\
\hline
\end{tabular}

de DAP, mas a classe DAP $\geq 50 \mathrm{~cm}$ apresenta sempre um ICM maior do que o das outras duas classes.

Essa evidência foi comprovada usando a análise de variância para comparar os ICMs médios das três classes de diâmetro. O resultado é apresentado na tabela 5 , indicando que pelo menos tendo principalmente maior oferta de luz . Outra explicação para esse fato é a consideração feita por Felippe (1979), em relação a maturidade dessas árvores: que uma vez já estabelecidas, teoricamente, a distribuição de energia pode ser direcionada, a fim de garantir biomassa lenhosa e sua fixaçāo ao solo.

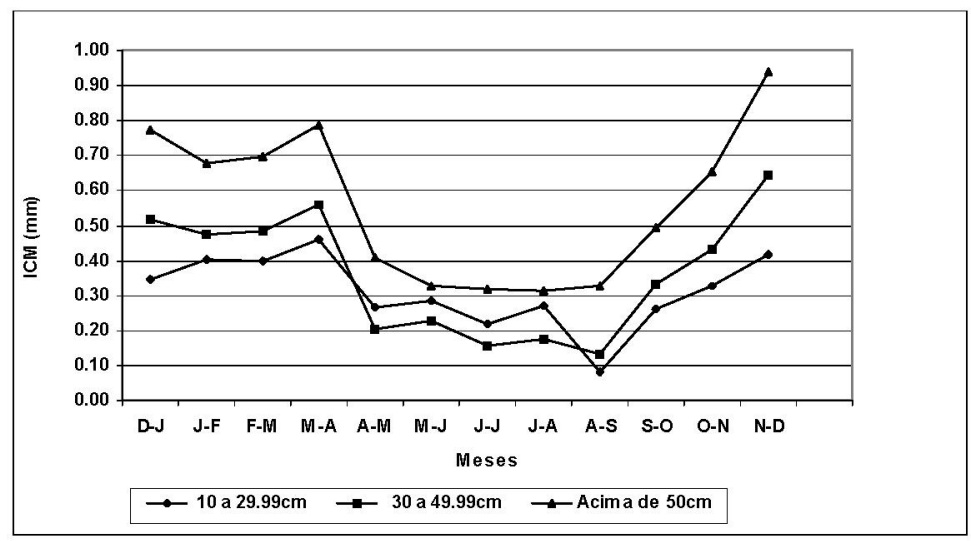

Figura 3. Curva de Incremento Corrente Mensal $(\mathrm{mm})$ nas diferentes classes de diâmetro 


\section{Comparação de médias entre transectos}

Testando a hipótese de que não há diferença de ICM em DAP entre os dois passar do tempo, são apresentados na Tabela

8. Essa análise foi executada de acordo com von Ende (1993), tendo o tempo (12 meses do ano

Tabela 6. Anova - Variação ICM entre e dentro dos transectos.

\begin{tabular}{lccccc}
\hline Fonte de variação & $\mathrm{SQ}$ & $\mathrm{GL}$ & $\mathrm{MQ}$ & $\mathrm{F}$ & $\mathrm{P}$ \\
\hline Fonte de variação & 0.00188 & 1 & 0.00188 & 0.65937 & 043260 \\
Entre transectos & 0.00054 & 2 & 0.00027 & 0.09477 & 0.91026 \\
Dentro dos trans. (CT) & 0.00163 & 2 & 0.00081 & 0.28617 & 0.75612 \\
Erro & 0.03417 & 12 & 0.00285 & & \\
\hline
\end{tabular}

transectos, verificou-se que, segundo a ANOVA (Tabela 6) ela é verdadeira não havendo diferença estatisticamente significativa entre os dois transectos $(\mathrm{p}=0,92)$.

Os dois transectos possuem, no solo, características físicas e químicas bem definidas e distintas, considerando a topografia (Ferraz et al., 1998). Entretanto, as observaçōes realizadas
2000) como parcelas subdivididas e considerando apenas a circularidade das matrizes como restrição à validade da ANOVA de parcelas repetidas.

Segundo ainda von Ende (1993), o valor de $\mathrm{F}$ deve ser corrigido, usando os fatores de Greenhouse-Geisser (G-G) ou Huynh-Feldt

Tabela 7. Análise de variância para as variáveis $\mathrm{ICM}(\mathrm{mm})$ entre e dentro das classes

\begin{tabular}{lcclccc}
\hline Fonte de variação & $\mathrm{SQ}$ & $\mathrm{gl}$ & $\mathrm{MQ}$ & $\mathrm{F}$ & Valor P & F crítico \\
\hline Entre topografias & 0.246933 & 5 & 0.049 & 1.56588 & 0.18196 & 2.3538 \\
Dentro das topografias & 2.081585 & 66 & & & & \\
Total & 2.328518 & 71 & & & & \\
\hline
\end{tabular}

neste estudo, demonstram uma forte semelhança no crescimento da vegetação dos dois transectos.

Comparação de médias entre as classes topográficas

Para testar a hipótese de que não há diferença de incremento corrente mensal (ICM) em DAP (mm) entre as três classes topográficas, compararam-se suas médias, obtendo resultados descritos na Tabela 7 , verificando-se que não há diferença estatisticamente significativa ( $\mathrm{p}=$ $0,05)$ entre as classes topográficas.

Os resultados da análise de variância (ANOVA) executada para verificar se as variações do incremento são devidas às classes topográficas e/ou classes de diâmetro, com o
(H-F), para inferir sobre cada fonte de variação, quando medidas repetidas são tomadas nos mesmos indivíduos ou unidades experimentais. Se o valor de G-G é menor do que H-F, o G-G tende a ser mais conservador do que H-F; por essa razão, é mais seguro fazer inferências com base em $\mathrm{G}-\mathrm{G}$.

Dessa maneira, apenas a variação do ICM em função do tempo é altamente significativa $(p=0,000)$, ou seja, o incremento varia com o passar do tempo, mesmo considerando o $\mathrm{F}$ não corrigido e $\mathrm{H}-\mathrm{F}$. A interação mês e classe de diâmetro é apenas significante ao nível de $10 \%(\mathrm{p}=0,079)$, mas não a $5 \%$, ou seja, as evidências não são fortes 
suficientes para afirmar categoricamente que o ICM varia de acordo com CD com o passar do tempo. Nesse caso, se fosse considerado $F$ não corrigido, a conclusāo poderia ser diferente e o nível de confiança passaria de 0,079 para 0,017 (quase significante ao nível de 1\%).

Da mesma forma, há fraca evidência ( $\mathrm{p}=$ confiabilidade das medições depende de considerações sobre a forma do caule, a fenologia da espécie e a ocorrência no fuste de infestações de cipós ou parasitas. As bandas usadas neste trabalho medem, no máximo, $20 \mathrm{~mm}$ de expansāo em circunferência. Portanto, quando atingem esse limite em menos de um ano, precisam ser trocadas;

Tabela 8. Análise de Variância para ICM entre e dentro $C T$, ICM em DAP (mm) e classes topográficas (CT) e classes de diâmetro (CD), com o passar do tempo.

a) Entre classes:

\begin{tabular}{lcrlcc}
\hline Fontes de variação & $\mathrm{GL}$ & $\mathrm{SQ}$ & $\mathrm{MQ}$ & $\mathrm{F}$ & $\mathrm{P}$ \\
\hline $\mathrm{CT}$ & 2 & 0.064 & 0.032 & 0.053 & 0.949 \\
$\mathrm{CD}$ & 2 & 3.258 & 1.629 & 2.690 & 0.086 \\
$\mathrm{CT}^{*} \mathrm{CD}$ & 4 & 0.180 & 0.045 & 0.074 & 0.989 \\
Erro & 27 & 16.352 & 0.606 & & \\
\hline
\end{tabular}

b) Dentro das Classes (parcelas subdivididas de acordo com o tempo):

\begin{tabular}{lccccccc}
\hline Fontes de variação & $\mathrm{GL}$ & $\mathrm{SQ}$ & $\mathrm{MQ}$ & $\mathrm{F}$ & $\mathrm{P}$ & $\mathrm{G}-\mathrm{G}$ & $\mathrm{H}-\mathrm{F}$ \\
\hline Mês & 11 & 11.675 & 1.061 & 19.306 & 0.000 & 0.000 & 0.000 \\
Mês & 22 & 0.575 & 0.026 & 0.980 & 0.980 & 0.886 & 0.940 \\
Mês $^{*} \mathrm{CD}$ & 22 & 2.166 & 0.098 & 0.017 & 0.017 & 0.079 & 0.040 \\
Mês & 44 & 2.991 & 0.068 & 0.157 & 0.157 & 0.246 & 0.200 \\
Erro & 297 & 16.328 & 0.055 & & & & \\
\hline
\end{tabular}

0,886) de significância da interaçāo mês e classe topográfica, ou seja, o ICM nāo tem nenhuma relação com a classe topográfica, com o passar do tempo. Usando os níveis de confiança clássicos (1\% ou 5\%), pode-se afirmar que, estatisticamente, não há nenhuma interação entre tempo, classe topográfica e classe de diâmetro porque o $G-G$ da interação é igual a 0,246 .

\section{CONCLUSÃO}

A utilização de bandas dendrométricas para monitorar o crescimento da árvore e do povoamento florestal, é prática e eficiente. Em modelagem e simulação da dinâmica da floresta, manejada ou não, quando informações básicas mais precisas são necessárias para alimentar os modelos estatísticos, as medições com bandas tornam-se mais importantes ainda. A trocar significa ter que esperar o tempo de ajuste da banda ao fuste.

Nos últimos 20 anos, a precipitação média nas proximidades da área de estudo foi de $2.610 \pm 124 \mathrm{~mm}(\mathrm{p}=0,05)$. Na área de estudo, no ano de 2000, a precipitaçāo total foi de $3.491 \mathrm{~mm}$. Portanto, o ano coberto por este estudo é um ano atípico do ponto de vista de precipitaçāo, com precipitaçāo anual caindo fora do intervalo de confiança da região.

O incremento em diâmetro varia muito $(\mathrm{p}$ $=0,000)$ de acordo com o mês do ano, ou seja, quanto maior é a precipitação do mês, maior é o incremento. Há também um sinal claro, mas não muito forte $(p=0,079)$, da interação mês e classes de diâmetro, ou seja, as árvores da maior classe de diâmetro (DAP $\geq 50 \mathrm{~cm}$ ) tendem a crescer mais do que aquelas das outras duas classes 
menores, conforme aumenta a precipitação mensal. No entanto, o sinal da interação mês, classe topográfica e classe de diâmetro é muito fraco $(\mathrm{p}=0,246)$, ou seja, é difícil distinguir a influência dos transectos, com o passar do tempo, ao crescimento individual das árvores. Por último, o sinal da interação mês e classe topográfica praticamente inexiste $(\mathrm{p}=0,886)$, ou seja, o padrão de crescimento individual é o mesmo no platô, encosta e baixio.

A média do incremento anual em circunferência, considerando todas as 272 árvores monitoradas, foi de $1,64 \pm 0,21 \mathrm{~mm}(\mathrm{p}=$ $0,05)$, ficando dentro do intervalo dos incrementos obtidos no BIONTE (área praticamente contígua à área de estudo) e FLONA Tapajôs (Santarém, Pará), que é de 1,5 a 2,0 $\mathrm{mm}$ por ano.

Algumas espécies listadas, utilizadas no manejo florestal da Coordenaçāo de Pesquisas em Silvicultura Tropical do INPA, como tachi vermelho (Sclerolobium setiferum Ducke), sucupira chorona (Andira micrantha Ducke), guariúba (Clarisia racemosa Ruiz \& Pav.), matamatá amarelo (Eschweilera coriacea (DC.) Mart. ex Berg.) e angelim rajado (Zygia racemosa (Ducke) Barneby \& J.W. Grimes) tiveram incremento anual em diâmetro igual a zero.

Por outro lado, várias espécies listadas tiveram incrementos anuais bem superiores aos incrementos médios estimados para a região amazônica, como tanimbuca (Buchenavia parvifolia Ducke), pau-marfim (Aspidosperma desmanthum Müll. Arg.), cardeiro (Scleronema micranthum Ducke), cumaru (Dipteryx odorata (Aubl.) Wild.), angelim pedra (Dinizia excelsa Ducke), marupá (Simarouba amara Aubl.), tachi vermelho (Sclerolobium setiferum Ducke), morototó (Schefflera umbrosa Frodin) e dois indivíduos de matamatá (Eschweilera coriaceae (DC) Mart. ex Berg.) e dois de louro-fofo (Ocotea aciphylla (Nees) Mez). Os incrementos em diâmetro para essas espécies variaram de 5,28 a $11,41 \mathrm{~mm}$ por ano, sendo igualmente superiores aos incrementos de espécies desenvolvidas em áreas sob manejo florestal, nos sítios do BIONTE e FLONA Tapajós, que não passam de $4 \mathrm{~mm}$ por ano.

\section{BIBLIOGRAFIA CITADA}

Bower, D.R.; Blocker, W.W. 1966. Accuracy of bands and tape for measuring diameter increments. J. For. 64: 21-22.

Cameron, R.J.; Lea, R. 1980. Band dendrometers or diameter tapes? J. For. 78: 277-278.

Chim, L.T.; On, W.F. 1973. Density, recruitment, mortality and growth of Dipterocarp seedlings in virgin and logged-over forests in Sabah. In: The Malasyan Forester. Vol. 36, no.1, p. 3-15.

Clark, D.B.; Clark, D.A. 1987. Population Ecology and Microhabitat Distribution of Dipteryx panamensis, a Neotropical Rain Forest Emergent Tree. Biotropica 19 (3) 236-244.

Clack, D.B.; Clark, D.A. 1999. Assessing the Growth of Tropical Rain Forest Trees: Issues for Forest Modeling and Management. Ecological Application, 9(3):981-997.

Condit, R.; Hubbel, S.P.; Foster, R.B. 1995. Demography and harvest potential of latin american timber epecies: data from a large, permanent plot in Panama. Journal Tropical Forest Science 7(4): 599-622.

Conner, W.H.; Day, J.W. Jr. 1992. Diameter growth of Rhodium distichum (L.) Rich. and Nyssa aquatica L. from 1979-1985 in four Louisiana swamp stands. Am. Midl. Nat. 127: 290-299.

Day, F.P. Jr. 1985. Tree growth rates in the periodically flooded Great Dismal Swamp. 
Castanea, 50: 89-95. In: Keeland, B.D. e Sharitz R.R. 1993. Accuracy of tree growth mensurements using dendrometer bands. Can. J. for Res. Vol. 23:2454-2457.

Felippe, G.M. 1979. Desenvolvimento dos Vegetais. In: Fisiologia Vegetal. Editora Pedagógica, v.2, capítulo 1, segunda edição. São Paulo, p.1-37.

Ferraz, J.; Ohta, S.; Salles, P.C. 1998. Distribuição dos Solos ao Longo de Dois Transectos em Floresta Primária ao Norte de Manaus (AM). Em: Pesquisas Florestais para Conservação da Floresta e Reabilitação de Áreas Degradadas da Amazônia. N. Higuchi, M.A.A. Campos, P.T.B. Sampaio e J. dos Santos (editores). pp.111-143.

Ferri, M.G. 1979. Fisiologia Vegetal. Editora Pedagógica, v.2, segunda edição. São Paulo, $401 \mathrm{p}$.

Finegan, B.; Camacho M.; Zamora, N. 1999. Diameter Increment Patterns among 16 Tree Species in a Logged and Silviculturally Treated Costa Rican Rain Forest. Forest Ecology and Management, 121 (3):159-176.

Freund, J.E.; Walpole, R.E. 1987. Mathematical Statistics. Prentice-Hall, Inc., EUA. $4^{\text {a }}$ edição. 608 p.

Hall, R. C. 1944. A vernier tree growth band. J. For. 42: 742-243.

Herwitz, S.R.; Young, S.S. 1994. Mortality, Recruitment, and Growth Rates of Montane Tropical Rain Forest Canopy Trees on Mount Bellenden-Ker, Northeast Queensland, Australia. Biotropica, 26(4):350-361.

Higuchi, N.; Jardim, F.C. da S.; Santos, J. dos; Barbosa, A. 1985. Bacia 3 - Inventário Florestal Comercial. Acta Amazonica 15(3-4):327-369.
Higuchi. N.; Santos.J. dos; Ribeiro. R.J.; Freitas, J.V.; Vieira, G.; Cöic, A. \& Minette, L.J. 1997. Crescimento e Incremento de uma Floresta Amazônica de Terra-Firme Manejada Experimentalmente In: Biomassa de Nutrientes Florestais. INPA/DFID. pp. 89-132.

Higuchi, N.; Santos, J. dos; Vieira, G.; Ribeiro, R.J.; Sakurai, S.; Ishizuka, M.; Sakai, T.; Tanaka, N.; Saito, S. 1998. Análise Estrutural da Floresta Primária da Bacia do Rio Cuieiras, ZF-2, Manaus-AM, Brasil. In: Pesquisas Florestais para Conservação da Floresta e Reabilitação de Áreas Degradadas da Amazônia. N. Higuchi, M.A.A. Campos, P.T.B. Sampaio e J. dos Santos (editores). pp.51-81.

Hubbell, S.P.; Foster, R.B.; O'Brien, S.T.; Harms, K.E.; Condit, R.; Wechsler, B.; Wright, S.; Loo de Lao, S. 1999. Light-Gap Disturbances, Recruitment Limitation, and Tree Diversity in a Neotropical Forest. Science, vol. 283, pp.554-557.

Keeland, B.D.; Sharitz, R.R. 1993. Accuracy of tree growth measurements using dendrometer bands. Can J. For. Res. Vol.23:2454-2457.

Lea, R.; Tierson, W.C.; Leaf, A.L. 1979. Growth responses of northern hardwoods to fertilization. For. Sci. 25: 597-604.

Leslie, A.J. 1987. The Economic Feasibility of Natural Management of Tropical Forests. In: Natural Management of Tropical Moist Forests: Silvicultural and Managent Prospects of Sustained Utilization. Edited by F. Megen e J.R. Vincent. Yale University Press. Vincent. Pp.178-198.

Manokaran, N. e K.M. Kochummen. 1993. Tree 
Growth in Primary Lowland and Hill Dipterocarp Forests. J.of Tropical F. Science, $6(3): 332-345$.

Milton, K.; Laca, E.A.; Demment, M.W. 1994. Successional Patterns of Mortality and Growth of Large Trees in Panamanian Lowland Forest. J. of Ecology, 82(1):79-87.

Poels, R.L.H.; de Graaf, N.R.; Wirjosentono, J. 1998. Growth and Mortality of Trees after Various Experimental Silvicultural Treatments for Natural Regeneration in Suriname. Agricultural University Wageningen, Hinkeloord Report $\mathrm{n}^{0} 25$.

Ribeiro, M. de N.G.; Adis, J. 1984. Local rainfall variability - a potential bias biological studies in the Central Amazon. Acta Amazonica, v.12, n0 14, p. 159-174.

Santos, H.M. 1968. Balanço Hídrico de Manaus, Amazonas: INPA. 8p.

Silva, R.P. da. 2001. Padrões de Crescimento de Árvores que Ocorrem em Diferentes Toposseqüências na Região de Manaus. Dissertação de Mestrado, INPA-CFT, 60p.

Silva, J.N.M., J.O.P.; Carvalho, J.C.A.; Lopes, R.P.; Oliveira e L.C. Oliveira. 1996. Growth and Yield Studies in the Tapajós Region, Central Brazilian Amazon. Comm. Forestry Review, 75(4):3325-329.

Tabuchi, R. \& Takahashi, K. 1998. The Development of a New Dendrometer and its Application to Deciduous Broadleaf Tree Species in Hokkaido, Northern Japan. Journal of Sustainable Forestry, 6(1/2):23-34.

Tribuzy, E. S. 1998. Metabolismo do Carbono e do Nitrogênio em Plantas de Ingá (Ingá vera Willd.) Submetidas a diferentes Alturas e Tempos de Submersão. Dissertação de mestrado, UFLA-Lavras, 64 p.

Turner, I.M. 1990. Tree Seedling Growth and Survival in a Malaysian Rain Foest. Biotropica, 22(2):146-154.

von Ende, C.N. 1993. Repeated-Measures Analysis: Growth and Other Time-Dependent Measures. Em: Design and Analysis of Ecological Experiments, editado por S.M. Scheiner e J.Gurevitch. Chapman \& Hall. pp. 113-137.

Wadsworth. F.H. 1987. Applicability of Asian and African Silviculture Systems to Naturally Regenerated Forests of the Neotropics. In: Natural Management of Tropical Moist Forests: Silvicultural and Management Prospects of Utilization. Edited by F. Mergen e J.F. Vincent. Yale University Press. pp. 94 111.

Walter, H. 1979. Vegetation of the Earth and Ecological Systems of the Geo-Biosphere. Springer-Verlag. New York. $274 \mathrm{p}$.

Weiss, N.; Hassett, M. 1982. Introductory Statistics. Addison-Wesley Publishing 651p.

\section{AGRADECIMENTOS}

Agradecemos aos projetos Jacaranda (MCT-INPA e JICA) e Piculus (MCT-FINEP e CCE), pelo apoio de coleta de dados; ao CNPq pelas bolsas DCR da primeira e da quinta autora e pela PQ do último autor; ao PCI-MCT pela bolsa do sexto autor.

Submetido à publicação: 16/08/2001

Aceito: 05/07/2002 